\title{
Marketing de los servicios bancarios en el ambiente competitivo de las sucursales de BANPRO y el BDF de la ciudad de Estelí, período 2014-2015
}

Orlando Zeledón Zeledón²

\section{RESUMEN}

Esta investigación, analiza el mercado de los servicios bancarios en el entorno competitivo de las sucursales de BANPRO y el BDF de la ciudad de Estelí, durante el período comprendido del 2014 al 2015. Para ello, se tomó en cuenta que la intermediación financiera que realizan estos bancos es muy importante para determinar el nivel de competitividad que tienen en el mercado bancario, a su vez se compararon entre si los servicios que ofrecen estas sucursales para establecer que banco brinda mejores productos y servicios que contribuyen en dar una mejor atención al público y finalmente se propusieron estrategias que apoyen al mejoramiento de la posición competitiva de estos bancos en relación a la oferta de nuevos servicios para satisfacer las necesidades de los clientes. Los resultados muestran que la intermediación financiera, da un aporte sustancial al empoderamiento competitivo de estos bancos en el mercado, además que los servicios ofrecidos por estas entidades son diversificados, pero aún deben de diseñar nuevos productos debido a la bancarización que está presente en el sector financiero, así mismo se propuso el establecimiento de nuevas estrategias que son de mucha utilidad para competir de una manera sana en el mercado bancario.

Palabras clave: Intermediación financiera, servicios bancarios, estrategias competitivas.

Recibido: 29 de febrero de 2016

Aceptado: 13 de junio de 2016

1 Este artículo hace referencia a la investigación titulada Marketing de los servicios bancarios en el ambiente competitivo de las sucursales de BANPRO y el BDF de la ciudad de Estelí, período 2014-2015 para optar al título de Máster en Gerencia Empresarial en el año 2015 por la UNAN- Managua, FAREM-Estelí.

2 Docente titular de la Universidad Nacional Autónoma de Nicaragua / Facultad Regional Multidisciplinaria. UNAN Managua / FAREM Estelí. Correo electrónico: orlanzelz@gmail.com 


\title{
Marketing of the banking services in the competitive environment of the branches of BANPRO and BDF in Estelí, 2014-2015
}

\begin{abstract}
This research analyzes the market of the banking services in the competitive environment of the branches of BANPRO and BDF, in Estelí, during the period of 2014-2015. For this purpose, it was taken into account that the financial intermediation is important to determine the level of competitiveness of the banking market. At the same time, the services offered by such branches were compared, in order to determine which bank offers best products and services that contribute to a better attention to the society. Finally, strategies aimed to the improvement of the competitive position of the banks were proposed. The main results reveal that the financial intermediation contributes to the competitive empowerment of the banks in the market. Even though the services offered by the branches are diversified, they must design new products due to the bancarization of the financial sector. Likewise, the establishment of new strategies to compete in a healthy way in the banking market was proposed.
\end{abstract}

Keywords: Financial intermediation, banking services, competitive strategies. 


\section{INTRODUCCIÓN}

El servicio al cliente constituye el punto que marca la diferencia entre empresas que venden productos $\mathrm{y} / \mathrm{o}$ servicios similares, al punto de ser un componente principal para el éxito o fracaso de una institución. En lo que respecta a la Banca Comercial, el servicio al cliente constituye una estrategia de mercado indispensable para el logro de los objetivos organizacionales. Los bancos que sobresalen, adoptan modelos orientados directamente a la satisfacción del cliente, el cual depende de la amabilidad, confiabilidad y empatía que pueda recibir de los empleados, pero fundamentalmente de la seguridad y prontitud en la obtención de información $\mathrm{y}$ datos que generen opciones para la resolución de sus transacciones financieras.

Sustentando lo antes mencionado, dicha investigación tiene mucha relevancia para tener conocimiento e información sistematizada sobre la oferta de los servicios y productos ofrecidos por las sucursales bancarias de BANPRO y el BDF en la ciudad de Estelí que contribuyen al desarrollo de las actividades económicas de la misma, al hacer uso de la intermediación financiera, a través de los depósitos y créditos que realizan estos bancos a los diferentes sectores productivos en la región.

Se narra sobre los servicios y productos que ofrecen estas sucursales, tomando en cuenta su diversificación, esto con el fin de mejorar la calidad del servicio para ser de éste, más eficiente para la población, principalmente para aquellos que acuden a estas instituciones a realizar algún tipo de transacción, lo que viene mejorar la atención a los clientes, esto con el fin de optimizar los beneficios tanto para los usuarios como para los bancos a través del proceso de intermediación que tiene cada entidad bancaria.

Se presenta un análisis comparativo de los servicios y productos que ofrecen estos bancos para determinar la posición competitiva que tienen en el mercado financiero, a su vez se elabora una propuesta de estrategias de mercado para mejorar la competitividad en el servicio brindado por estas entidades al público en general, por lo que permitirá a cada institución lograr un mejor posicionamiento en el mercado tomando en cuenta la competencia.

\section{MATERIALES Y MÉTODOS}

Esta investigación es de tipo aplicada, puesto que, para la obtención de la información, se dio a través de la implementación de instrumentos de recolección de datos de una población real que visitan estos bancos y hacen uso de los servicios que ofrecen a la población, además tiene un enfoque cualitativo, cuantitativo y transversal porque explica e interpreta los resultados en un período de tiempo ya establecido, a su vez se hace uso del muestreo probabilístico dentro del cual está el aleatorio simple.

Para la recolección, procesamiento y análisis de los datos obtenidos, se tuvo previsto la obtención de la información a partir de fuentes primarias directas por lo que se realizaron entrevistas a funcionarios que laboran para estas instituciones, y la aplicación de una encuesta a los usuarios y clientes que visitan estos bancos que hacen uso de los servicios que ofrecen al público en general, por último se hizo el procesamiento de este instrumento a través del programa IBM SPSS, el que generó datos cuantitativos por medio de tablas de frecuencias y gráficos, la cuales fueron de mucha utilidad para procesar y sustentar la información de la investigación.

\section{RESULTADOS Y DISCUSIÓN}

Intermediarios financiera en relación a la oferta de los servicios brindados por estas sucursales bancarias.

La intermediación financiera, como actividad esencial de estas entidades, han hecho uso de estrategias 
bien definidas para captar mayor número de cuenta habientes, lo que ha favorecido a cada institución, obtener mayores depósitos y disponerlos en forma de créditos a los diferentes sectores económicos del país.

Para sustentar lo antes mencionado, se presentan dos cuadros comparativos, que determinan el comportamiento de estos bancos en la captación de ahorro y en la colocación de los fondos al crédito. Para la elaboración de estos cuadros se necesitó de datos cuantitativos suministrados por la Superintendencia de Bancos y de otras Instituciones Financieras de Nicaragua, de las sucursales de BANPRO y el BDF que operan en nuestro país.

Cuadro $\mathrm{N}^{\circ}$ 1: Depósitos en moneda nacional y extranjera PARTICIPACIÓN DE MERCADO - DEPÓSITOS (Miles)

\begin{tabular}{lrrrr}
\hline \multirow{2}{*}{ Entidad Bancaria } & \multicolumn{2}{c}{ Diciembre - 2014 } & \multicolumn{2}{c}{ Septiembre - 2015 } \\
\cline { 2 - 5 } & Monto en C\$ & Monto en \$ & Monto en C\$ & \multicolumn{1}{c}{ Monto en \$ } \\
\hline BANPRO & $11,526,567.8$ & $1,184,946.3$ & $12,545,920.6$ & $1,117,439.2$ \\
BDF & $2,053,835.7$ & $328,169.3$ & $2,021,919.3$ & $368,513.8$ \\
\hline
\end{tabular}

Fuente: Elaboración propia con datos de la Superintendencia de Bancos de Nicaragua

Cuadro N²: Créditos en moneda nacional y extranjera

PARTICIPACIÓN DE MERCADO - CRÉDITOS (Miles)

\begin{tabular}{|c|c|c|c|c|}
\hline \multirow{2}{*}{ Entidad Bancaria } & \multicolumn{2}{|c|}{ Diciembre - 2014} & \multicolumn{2}{|c|}{ Septiembre - 2015} \\
\hline & Monto en C\$ & Monto en \$ & Monto en C\$ & Monto en \$ \\
\hline BANPRO & $1,670,205.8$ & $933,813.40$ & $1871,058.20$ & $1,037,820.9$ \\
\hline BDF & $519,707.60$ & $421,372.70$ & $549,640.10$ & $479,945.50$ \\
\hline
\end{tabular}

Fuente: Elaboración propia con datos de la Superintendencia de Bancos de Nicaragua

Como se aprecia de manera comparativa, en el período comprendido entre los años del 2014 y el 2015, la sucursal bancaria de BANPRO se ha posesionado mejor en el mercado, en cuanto a los depósitos y la cartera crediticia destinada a los sectores productivos en comparación al BDF; esto se debe a que los depósitos en moneda nacional y extranjera están más diversificados en nuevas cuentas de ahorro, y cuentas a plazo con atractivas tasas de interés así como una mayor cobertura que tiene el banco en la región, contando con nuevos sistemas tecnológicos de depósitos conocidos como Agente BANPRO, el cual permite efectuar depósitos, retiros, pagos de servicios básicos en establecimientos comerciales que están cerca de su casa o en su barrio y en lugares donde el sistema bancario aún no tienen presencia.

En cuanto al sector crediticio de BANPRO, está destinando más a los préstamos a los sectores de la banca empresarial y entre las carteras más importantes están la de consumo y la comercial las que a su vez incluyen las industrias; pequeñas y medianas empresas además del sector agrícola y lo más novedoso es que a finales del año 2014 ha promovido proyectos denominados "líneas verdes" con el objetivo de generar financiación con propósitos ambientalistas relacionados con el uso eficiente de la energía eléctrica y el cuido del medio ambiente.

En resumen, esto explica que la entidad bancaria BANPRO presenta crecimientos muy significativos respecto a la intermediación financiera relacionada con depósitos y créditos que ha realizado el BDF; sin embargo, se puede apreciar que este banco, siendo un banco joven y con menor cobertura presenta indicadores muy positivos, tanto en depósitos como en préstamos sobre una base sólida que tiene en el mercado financiero. 
Análisis de los servicios bancarios que ofrecen estas sucursales en dar una mejor atención a la población de la ciudad de Estelí

Se procedió la aplicación de encuestas a usuarios y clientes que visitan estos bancos, así como entrevistas a funcionarios que laboran para estas instituciones, esto con el fin de determinar qué servicios y productos dan mejor atención y mayor satisfacción a la población que acude al banco de su preferencia, por lo que se llegaron a los siguientes resultados.

La mayoría de la población que visitan estas entidades, según encuesta, están entre los rangos de edades de 20 - 30 años, para el caso de BANPRO corresponde aproximadamente el $70 \%$ y para el BDF el $67 \%$, por lo cual es bastante similar para ambas sucursales. Esto significa de manera muy relativa para los dos bancos una oportunidad dado que, si los clientes están en este segmento de mercado joven, la edad da la pauta para la formulación de nuevos productos dirigidos a este grupo de interés.

Así mismo de manera muy similar para ambas sucursales, se tomó en cuenta el nivel académico de los usuarios, siendo el más representativo la preparación universitaria, por lo que esto es un punto muy interesante, puesto que este tipo de clientes conoce lo relacionado con el funcionamiento de una entidad bancaria por lo que se vuelve un cliente más exigente e informado de los servicios y productos que ofrecen en el mercado, esta información es valiosa si se estima que cada una de estas entidades bancarias ofrece al mercado productos financieros similares, y al atender esta información se visualiza que se convierten en competidores directos por el segmento de mercado al que pertenecen sus clientes actuales.

Otro aspecto que se consideró tomar en cuenta fue la ocupación que tiene la población que suele visitar estas entidades, del total de encuestados para la entidad bancaria de BANPRO, el 61\% son asalariados y el 39\% corresponden a clientes que tienen negocio propio, para el BDF el 58\% corresponden a clientes asalariados y el $42 \%$ poseen negocio propio. Esto significa, que, para ambas entidades, de manera similar, la mayoría de los clientes y usuarios que los visitan son asalariados.

Sin embargo, es necesario destacar que, para ambos bancos, se tiene un peso bastante alto, aquellos clientes que tienen negocio propio, ya que a través de la entrevista que se les hizo a los funcionarios de cada banco, respondieron de manera análoga, que estos clientes llegan a solicitar créditos para ampliar sus negocios o bien acuden a realizar pagos o abonos de préstamos ya establecidos.

En términos generales, según los funcionarios entrevistados, relatan que es muy significativo que visiten estas sucursales, tanto asalariados como aquellos que tienen negocios propios, por lo que es de mucha importancia a la hora de tomar decisiones por parte del banco, puesto que son un segmento de la población que es estable, en edad laboral y con fuertes potenciales financieros.

Sobre la satisfacción de los clientes con la atención recibida, se aprecia de manera muy parecida tanto para BANPRO y el BDF que es calificada de ser muy buena, con porcentajes de $80 \%$ y $84 \%$ respectivamente, por lo que el personal que les atiende, según los encuestados, consideran que son amables, cortés y educados, así como competentes, esto muestra que para cada uno de estos bancos es una fortaleza, porque se le da una mejor imagen y proyección de la entidad que forman parte, así como del personal que trabaja en cada institución.

Sin embargo, al considerar el tiempo de espera para ser atendidos por el personal de servicios bancarios, según opinión de los usuarios, critican que es bastante lento en cada uno de los bancos, antes esta situación, al preguntarles a los funcionarios que laboran para estas instituciones, comentaron que para dar salida a 
esta problemática tanto en BANPRO como el BDF, abrieron una ventilla, en MULTICENTRO Estelí, con el objetivo de darles solución a los clientes y estos puedan ser atendidos de manera más rápida.

En relación a los servicios que ofrecen mejores beneficios para los clientes, se muestra que tanto para BANPRO y el BDF, del total de encuestados que corresponde el $60 \%$ y el $66 \%$ respectivamente para cada entidad, son las cuentas de ahorro. Para detallar más sobre este aspecto, la población prefiere hacer uso de este producto bancario por que ofrecen tasas de interés bastante atractivas, que se capitalizan diariamente según los depósitos que realicen, además porque poseen novedosos premios, promociones y rifas en diferentes épocas del año.

Con respecto a la bancarización en línea y de los productos adicionales que ofrece este novedoso servicio que brinda cada banco, se les preguntó a los clientes si hacían uso de él, los resultados muestran que del total de encuestados, para el caso de BANPRO el $70 \%$ de la población opina que no hace uso de este servicio, y un 30\% de la población respondió que sí lo hace frecuentemente, para la entidad financiera del BDF, respondió que un $79 \%$ no hace uso de este servicio tecnológico y solamente un $21 \%$ de los clientes afirmó que sí lo hace.

De lo anterior, se interpreta que, para ambas sucursales, los indicadores son muy similares, en cuanto a los porcentajes que no son muy favorables para cada banco. La mayoría de los usuarios y clientes, todavía no hacen uso de este novedoso servicio, aun cuando estos bancos invierten altas sumas de dinero en promocionar este servicio que es completamente moderno, por el cual se pueda acceder de manera fácil y segura para realizar cualquier tipo de transacción o transferencia desde un ordenador o celular, vemos que la población le genera mucha desconfianza en hacer uso de la bancarización en línea.
Otro aspecto muy relevante que se les preguntó a los clientes, es si debería contar el banco con un servicio adicional y actualmente éste no lo tiene, para el caso de BANPRO, solamente el 25\% de los encuestados señaló que sí deberían ofrecer o ampliar más servicios, para el caso del BDF el 59\% expresó que el banco debería ofrecer más y nuevos productos financieros.

Según el análisis de estos resultados, se observa una situación bastante diferente para cada banco, para el caso de BANPRO, se aprecia que la mayoría de los usuarios consideran que el banco no debería ofrecer servicios adicionales, en contraste con los clientes que visitan el BDF, sugieren que si deberían ampliar o diversificar más productos y servicios bancarios, debido a las exigencias que está teniendo la población hoy en día, en la búsqueda de servicios no tradicionales que sean cada vez más competitivos en relación a los que ofertan otros bancos.

Para sustentar lo antes mencionado, se les solicitó a los encuestados que expresaran de manera libre, sobre cuál es el servicio adicional que debería tener la entidad bancaria para dar respuesta a las exigencias y necesidades que requieren los clientes de estos bancos, para el caso de los usuarios que visitan la sucursal de BANPRO, aunque representan una minoría, sugieren que debe de haber más personal en servicios bancario en horas de mayor demanda y abrir más ventanillas en otras áreas comerciales de la ciudad de Estelí.

Para el caso del BDF, sugieren que deben de establecer más cajeros automáticos en los centros de mayor comercio y en los supermercados, atención con más personal en área de caja en horas de mayor afluencia por parte de la población, abrir más ventanillas en otras áreas de la ciudad, así como nuevas cuentas de ahorro para jóvenes y estudiantes, dar apertura a cuentas en euros, diversificar los servicios automatizados, ampliar más actividades financieras con negocios en los barrios, se mencionan los más relevantes. 


\section{Comparación de los servicios bancarios de estas instituciones en su entorno competitivo}

Ambas entidades, tienen servicios bastante comunes, como son cuentas de ahorro, depósitos a plazo, préstamos, operaciones internacionales, financiamiento de seguros, tarjetas de créditos, intermediaciones leasing y Factoring, entre otras. Estos servicios tienen bastante demanda por diferentes segmentos de la población, así como empresas públicas y privadas que tienen mucha confianza en depositar su dinero a estos bancos.

También, se observa que dentro de estos servicios que son similares, existen otros dentro de ellos, que se pueden consideran como particulares, es decir de otra forma como exclusivos, y estos son: las novedosas cuentas de ahorro, éstas se han diversificado de acuerdo a las necesidades y preferencia del cliente, ya que tienen beneficios diferenciados, como por ejemplo: permite aplicar líneas sobre giro mayor de los fondos invertidos, devengan intereses a través de inversiones nocturnas (over night), le da oportunidad a tener su tarjeta de crédito, se hacen promociones y rifas en diferentes temporadas del año, ganan puntos por compras en comercios afiliados, acceso a banca en línea, para hacer transferencias y pagos de otros servicios, entre otros.

En el caso de los préstamos, también estos bancos han diversificado esta línea, para ser más competitivos, algunos beneficios pueden ser: establecimiento de préstamos con respaldo de fondos que con los que el cliente posee en el banco, se dispone de financiamiento de manera inmediata para atender necesidades imprevistas o aprovechar oportunidades de inversión, tasa de intereses negociables de acuerdo al tipo de negocio que tiene el cliente, entre otras.

En el caso de los servicios tecnológicos, ninguno de los dos bancos se queda atrás, se observar que tanto BANPRO como el BDF han innovado sus servicios dentro de una nueva era que es conocida como bancarización, estos productos son diseñados para satisfacer los requerimientos y las necesidades de los clientes y los negocios, ya que desde la comodidad de su hogar o en su trabajo puede acceder a todos los servicios bancarios que ofrece cada banco para ejecutar cualquier transferencia que el cliente desee, de igual manera es una solución para aquellas pequeñas y medianas empresas que requieran de un medio de pago que facilite sus operaciones y reduzca costos en transacciones por lo que es completamente seguro, fácil y totalmente en línea.

Una vez analizados y comparados los servicios que ofrecen BANPRO y el BDF, es necesario clasificar estos servicios según la categorización que establecen algunos observadores de los mercados financieros, a través de tres categorías, las cuales son muy útiles para determinar la competitividad que tiene cada banco en el mercado.

En la primera categoría que trata de los servicios bancarios de ampliación del mercado y manejo de riesgo, se incluyen principalmente aquellos servicios que aumentan los recursos monetarios en los bancos, lo que a su vez el banco los diversifica para mejorar y minimizar los riegos para los clientes. Para BANPRO y BDF, los servicios que estarían en esta primera categoría serían las novedosas cuentas de ahorro, como vemos tanto par BANPRO y BDF han diversificado las cuentas de ahorro, para atraer más clientes; sin embargo, desde el punto de vista competitivo, BANPRO está mejor posesionado que el BDF en cuanto a la cantidad de nuevas cuentas de ahorro que ha abierto para los clientes. Esto se puede comprobar verificando los cuadros comparativos que se muestran al inicio relacionados a la intermediación financiera sobre los depósitos que se han hecho en cada banco

La segunda clasificación hace referencia a los servicios bancarios de transferencia de riesgos y generación de liquidez, son aquellos servicios que 
generan rentabilidad al banco al transferir los depósitos y ahorros a los sectores productivos, es decir que esta segunda categoría hace referencia a los préstamos de igual manera a otros servicios que le crean liquidez al banco.

Tanto para BANPRO y el BDF, en cuanto al servicio de los préstamos, está bien estructurada para cada uno de ellos. De la misma manera, BANPRO está mejor ubicado que BDF en cuanto a esta actividad. Esta afirmación se puede verificar en los cuadros que están al inicio, relacionado a la intermediación financiera que se explica sobre la cartera crediticia por parte de cada uno de las sucursales.

La tercera y última clasificación detalla los servicios tecnológicos bancarios, explicados como todos aquellos servicios auxiliados con el uso de la TIC e internet, que han venido a revolucionar y dar una imagen completamente moderna a estas instituciones, en este caso tanto para BANPRO y BDF, no se quedan atrás en relación a los servicios tecnológicos, por lo que cada uno de ellos, está creando novedosos productos electrónicos para competir y ser más atractivo a sus clientes.

Para el caso de BANPRO, se aprecia que está mejor posicionado en cuanto a servicios tecnológico, al introducir desde el año 2014, el servicio de Agente BANPRO, que ha venido a mejorar la atención al cliente, según entrevista realizada a funcionario de esta institución, comentó que el banco tiene en varios establecimientos comerciales en la ciudad de Estelí, equipos electrónicos (ATM), es decir cajeros automáticos, donde los cuentahabientes pueden introducir sus tarjetas de crédito o débito para realizar retiro o depósito de dinero, pagar lo servicios básicos, recibir remesas, entre otros, además afirmó que con el sistema Agente BANPRO, el Banco de la Producción se coloca al frente de los demás bancos porque hace uso de un servicio alternativo para realizar transacciones bancarias, concluyó diciendo que es un sistema totalmente seguro y se puede realizar con la mayor tranquilidad, sin la necesidad de ir donde está el banco, ya que en muchos barrios de la ciudad, se están dando este servicio.

En el caso del BDF, el funcionario comentó que el banco aún no tiene este servicio diversificado como lo tiene BANPRO, aunque si argumentó que en la ciudad capital se tiene un servicio parecido llamado Punto XPRESS, pero que, en Estelí, todavía el servicio es muy limitado.

Sustentando lo antes mencionado en relación a la comparación y clasificación de los servicios y productos que ofrecen estas sucursales bancarias en su entorno competitivo, se proponen algunas estrategias de mercado que aporten a cada banco, brindar mejores servicios según las necesidades y exigencias que tengan los clientes, contribuyendo de esta a forma mantener una posición más competitiva en el sector financiero, el cual será abordado en el siguiente apartado.

\section{Propuesta de estrategias que contribuyan al mejoramiento de la posición competitiva en la oferta de servicios bancarios para BANPRO Y BDF}

Los continuos cambios que se dan en el entorno financiero están provocando modificaciones significativas en la estrategia de las entidades que operan en el mismo. El incremento de la competencia, el dinamismo de los mercados en cuanto a la exigencia de los consumidores, la progresiva desregulación y el proceso de globalización son entre otros el reflejo de las tendencias que se vienen produciendo en el sector bancario de nuestro país.

Ante este panorama, se presentan como posibles alternativas estratégicas para mantener una perspectiva de fortalecimiento y progreso en el nivel competitivo de estas dos sucursales que prestan servicios en la ciudad de Estelí, a continuación, se mencionan:

Primera estrategia: Calidad en el servicio y los productos que se ofrecen al público en general. 
Segunda estrategia: Fortalecimiento de políticas de innovaciones financieras y tecnológicas.

Tercera estrategia: Segmentación de grupos etarios en los clientes.

Cuarta estrategia: Establecimiento de alianzas con otras empresas de servicios especializados.

Quinta estrategia: Cultura de educación financiera a los usuarios y clientes de los bancos comerciales.

\section{CONCLUSIONES}

Al realizar el análisis de los resultados se concluyó lo siguiente:

a. La intermediación financiera seguirá siendo siempre un indicador muy importante para el crecimiento de las entidades bancarias, porque determinan el comportamiento que tienen los depósitos de los ahorrantes y que son canalizados por medio de la financiación hacia los sectores productivos, esto es un aporte sustancial al empoderamiento competitivo que tienen estas instituciones en el mercado bancario.

b. Se logró identificar que los servicios ofrecidos por BANPRO y el BDF al público en general que presentan mejores beneficios son las cuentas de ahorro por sus novedosos y atractivos premios y promociones que se hacen en diferentes épocas del año.

c. Con la introducción de nuevas tecnologías financieras por parte de estos bancos han constituido un importante soporte para el desarrollo de nuevos productos y servicios que son operados a través de plataformas web conocida como banca en línea, las cuales permiten al cliente una atención más rápida en tiempo real y a bajo costo; sin embargo a pesar de las grandes inversiones que hacen estos bancos, todavía es subutilizada por parte de la población por lo que les genera desconfianza al hacer uso de la bancarización electrónica. d. Es oportuno haber clasificado y agrupado los servicios y productos que ofrece estas entidades para ser analizarlos de manera comparativa y determinar su nivel competitivo, para dar nos cuenta que la sucursal bancaria de BANPRO está mejor posesionada financieramente que BDF en cuanto a servicios de ampliación de mercado y manejo de riesgo; transferencia de riesgo de precios y generación de liquidez y de manera similar en cuanto a los servicios tecnológicos bancarios que ofrecen a la población

e. Ante los continuos cambios que se están presentando en el entorno financiero debido a la globalización de los mercados, se están gestando modificaciones significativas por parte de cada uno de estos bancos en el establecimiento de nuevas estrategias, las cuales serán de mucha utilidad para competir de una manera sana en dicho sector con la captación de más clientes potenciales y de satisfacer sus necesidades

\section{RECOMENDACIONES}

Se recomienda tomar en cuenta los siguientes aspectos para la mejora permanente en la oferta de los servicios y productos que ofrecen estos bancos, entre las cuales se mencionan:

a. Deben de seguir diversificando los servicios y productos que ofrecen a la población, ya que no solo las cuentas de ahorro generan satisfacción, mucho de los usuarios que visitan estas sucursales son adultos jóvenes y en su mayoría profesionales, por lo que deberán promover otros servicios pensados en cada una de sus necesidades, por lo que estas son diferentes, y lo que se busca es fidelizarlo como nuevo y permanente cliente en dicha entidad.

b. Se les siguiere a estos bancos, que, así como han llegado a centros urbanos, no dejar al margen otros lugares del municipio donde no se han establecido y tener presente que muchos pobladores demandan de los servicios de los bancos, por lo que así se les ahorraría tiempo y dinero a los clientes 
al trasladarse a la ciudad para realizar sus diversas obligaciones y necesidades bancarias.

c. Se les recomienda a estas entidades bancarias seguir agotando recursos de convencimiento a la población para que haga uso de la bancarización relacionada a la banca en línea, además de hacerle saber que es un servicio fácil y muy seguro para realizar varias actividades financieras y servicios adicionales sin necesidad de llegar al banco.

d. Poner en práctica ciertas estrategias competitivas que les permitirán seguir manteniéndose en el mercado financiero ante los demás bancos que operan en la región.

\section{BLIOGRAFÍA DE REFERENCIA}

Albalá, M.A.(1998). El Sistema Bancario Nicaragüense en la década de los 90. Encuentro, 10-25.

Avendaño, N. (2014). Ranking de la Banca Comercial. Blog de Néstor Avendaño, 12-15.

Bancos, S. I. (2008). Ley general de bancos, intitutciones financiera y no financieras. Managua.

BCN. (2008). Intermedición al vencimiento. Managua

Daniel S. Behar. Metodolgía de la Investgición. México. 2008. Prentice Hall

Fonseca I, R. (2008). El Estado actual de la banca comercial nicaragüese. El Observador Ecónomico , 2656.
Frank J. Fabozzi, F. M. (2010). Mercado e Instituciones Financieras. México: Prentice Hall.

Hernández, Sampieri y Mendoza. (2003) Metodología de Investgación. México: Mc Graw Hill

Kotler, P., \& Armstrong, G. (2008). Fundamentos de Marketing. México: Pearson Educación de México, S.A de C.V.

Lambin, J. G. (2009). Dirección de Marketing. Gestión estratégica y operativa del mercado. México: Mc Graw Hill.

Lucydalia Blanca, W. A. (2013). Bancarizacion del gran desafio de los servicios bancarios. La Prensa, págs. 3-5.

Martinez Alonso, D. (2002). Servicios Bancarios, marcan la tendencia en los bancos. La tribuna, 1822.

Membreño, A. (12 de Agosto de 2014). Material de Planificación Estratégica. Esteli, Nicaragua.

Ricarod Guerrero, L. N. (8 de julio de 2013). Bancos diversifican su oferta de servicios bancario. Nuevo Darios, págs. 3,4.

Samaniego, J. D. (2008). Administración Financiera . En J. D. Samaniego, Administración Financiera (págs. 29,32). México : Mcgraw - Hill.

wwwbanpro.com

wwwbdf.com

wwwsiboif.com 